UDC 612.17

DOI: 10.21668/health.risk/2021.1.14.eng

Research article

\title{
COMBINATION OF HLA-DRB1 ALLELES AS A FACTOR CAUSING RISKS OF SPORADIC CONGENITAL HEART DEFECTS AND CONGENITAL MALFORMATIONS WITHOUT CHROMOSOME DISEASES
}

\author{
A.V. Shabaldin ${ }^{1,2}$, A.V. Tsepokina ${ }^{1}$, O.V. Dolgikh ${ }^{3}$, E.V. Shabaldina ${ }^{2}$, A.V. Ponasenko ${ }^{1}$ \\ ${ }^{1}$ Scientific Research Institute for Complex Issues of Cardiovascular Diseases, 6 Sosnovyi Blvd., Kemerovo, \\ 650002, Russian Federation \\ ${ }^{2}$ Kemerovo State Medical University, 22a Voroshilova Str., Kemerovo, 650056, Russian Federation \\ ${ }^{3}$ Federal Scientific Center for Medical and Preventive Health Risk Management Technologies, 82 Monastyrskaya \\ Str., Perm, 614045, Russian Federation
}

Congenital heart defects are anomalies that are becoming more and more frequent every year. Their specific weight is the highest among all the defects and malformations in fetus. Besides, children with sporadic congenital heart defects and malformations are still born rather frequently. We made an assumption that congenital heart defects (CHD) and congenital malformations (CM) were formed due to inflammatory process decompensation within «mother - fetus» system occurring in case of a conflict as per HLA between a semi-allogenic fetus and its mother's microenvironment. A risk of such a conflict might be associated with certain HLA combinations in parents' genotypes.

Our research goal was to reveal peculiarities of HLA-DRB1 alleles combinations in married couples who had children with sporadic CHD and CM without any chromosome diseases and to determine whether such peculiarities could cause risks of congenital anomalies.

We determined frequency of 14 alleles in HLA-DRB1 gene in all people who took part in the research.

Our research allowed establishing that parents whose children suffered from CHD more frequently had common $H L A-D R B 1 * 04$, female HLA-DRB1*07 with male HLA-DRB1*13, HLA-DRB1*17 and female HLA-DRB1*13 with male $H L A-D R B 1 * 14$. Children who suffered from CM more frequently had parents who were homologous as per HLA-DRB1*12, as well as with female HLA-DRB1*12 and male HLA-DRB1*01, HLA-DRB1*04, HLA-DRB1*13, and HLA-DRB1*15; this greater frequency was statistically significant. We also detected an authentic increase in frequency of HLA-DRB1*12 allele in children against their parents. Children with CM also had HLA-DRB1*12 allele statistically significantly more frequently than healthy children.

Peculiarities related to HLA-DRB1 alleles combination are genetic predictors of CHD and CM occurrence; their determination will allow minimizing risks of such disorders due to early diagnostics and timely prevention.

Key words: major histocompatibility complex, HLA-DRB1, alleles, congenital heart diseases, congenital malformations, risk factor, married couples, spouse compatibility.

The major histocompatibility complex (HLA in a human body) is a vital component in the immune systems of mammals (and people as well). A set of genes that are included into HLA is localized as per three classes (I, II, and III). The class II contains HLA-DR and HLA-DQ loci with their genes coding molecules that present endo- and exo-antigens to T-helper lymphocytes (of the 1,2, 3, 17 and 22 types). Through this phenomenon, they de-

(C) Shabaldin A.V., Tsepokina A.V., Dolgikh O.V., Shabaldina E.V., Ponasenko A.V., 2021

Andrey V. Shabaldin - Doctor of Medical Sciences, Associate Professor, Leading researcher at the Heart Defects Laboratory; Professor at the Department for Microbiology, Immunology, and Virology (e-mail: weit2007@yandex.ru; tel.: +7 (903) 907-51-97; ORCID: https://orcid.org/0000-0002-8785-7896).

Anna V. Tsepokina - Junior researcher at the Genome Medicine Laboratory (cepoav1991@gmail.com; tel.: +7 (950) 586-33-97; ORCID: https://orcid.org/0000-0002-4467-8732).

Elena V. Shabaldina - Doctor of Medical Sciences, Associate Professor, Head of the Otorhinolaryngology Department (e-mail: weit2007@yandex.ru; tel.: +7 (951) 163-90-11; ORCID: https://orcid.org/0000-0002-0450-2767).

Oleg V. Dolgikh - Doctor of Medical Sciences, Professor, Head of the Department for Immune-Biological Diagnostic Procedures (e-mail: oleg@fcrisk.ru; tel.: +7 (342) 236-39-30; ORCID: http://orcid.org/0000-0003-4860-3145).

Anastasia V. Ponasenko - Candidate of Medical Sciences, Head of the Genome Medicine Laboratory (e-mail: ponaav@kemcardio.ru; tel.: +7 (951) 591-05-50; ORCID: https://orcid.org/0000-0002-3002-2863). 
termine power and quality of immune responses to macro- and micro-ecology antigens [1]. Genes belonging to HLA I and II classes are highly polymorphic. Thus, according to HLA Alleles Numbers ${ }^{1}$ there are more than 2,500 alleles described at the moment for HLA-DRB1 only. HLA-DRB1*01, HLA-DRB1*03 (17), HLADRB1*04, HLA-DRB1*07, HLA-DRB1*11, HLA-DRB1*12, HLA-DRB1*13 and HLA$D R B 1 * 15$ alleles are evenly distributed within populations in the world [2-4]. It was revealed that alleles included into $H L A-D R B 1 * 03$ (17), HLA-DRB1*04, HLA-DRB1*05(11), and HLA$D R B 1 * 15$ groups had certain associations with immune system pathologies and reproductive losses $[5,6]$. It is assumed that there are several selective mechanisms in populations that are responsible for imposing limits on random inheritance and for controlling "pathologic» alleles within this or that population. Such selective mechanisms include, for example, negative-assortative mating, selection at gametogenesis level, selection in interactions as per HLA between a mother and a semi-allogenic embryo/fetus, as well as intensity of resistance or sensitivity to infectious and parasitic agents [7]. Let us note that ontogenesis gives this exact order of selection mechanisms.

Congenital heart diseases (CHDs) are a topical issue for public healthcare since their frequency is growing and they account for the biggest share among all malformations and development anomalies [8, 9]. Besides, children with sporadic congenital malformations and development anomalies (CMDAs) without any chromosome diseases are still born rather frequently. There is a well-grounded assumption that CHDs and CMDAs occur, among other things, due to decompensation with an inflammatory process within «mother - fetus» system that develops in case there is a conflict as per HLA between a semi-allogenic fetus and micro-environment inside a mother's body $[10,11]$. A risk of such a conflict can be associated with certain HLA combinations in parents' genotypes.

Bearing in mind that an association between certain alleles and HLA genotypes and immune-inflammatory diseases and reproductive losses, including congenital fetus/embryo malformations, has been proven [12, 13], we believe that a study on peculiarities of alleles belonging to a gene in the major histocompatibility complex (HLA) will allow determining how compatible parents are, hence, it will provide an opportunity to estimate a risk that a child will be born with congenital malformations and development anomalies thus resulting in reproductive losses.

Given that, our research goal was to determine peculiarities of HLA-DRB1 allele combinations in families who had children with sporadic congenital heart diseases and congenital malformations without chromosome diseases as well as a nature of their inheritance by these children.

Data and methods. Our research was accomplished at the Scientific Research Institute for Complex Issues of Cardiovascular Diseases, Kemerovo State Medical University and Kemerovo State University. The research was approved by the local ethical committee at the Scientific Research Institute for Complex Issues of Cardiovascular Diseases. All the participants gave their informed written consent to take part in the research.

The first test group was made up of 48 families with children in them (23 boys and 25 girls) being born with congenital heart diseases regardless of any chromosome disease. We examined case histories in this group and revealed that there were no congenital heart diseases either in mother's or father's ancestors. HLA-DRB1 allele combinations were calculated in families both regarding female alleles meeting with male ones and vice versa. Overall, we analyzed 384 combinations $(48 \cdot 8=384)$. This test group was created at the Scientific Research Institute for Complex Issues of Cardiovascular Diseases and the Kemerovo State Medical University.

The second test group consisted of 68 families with children having congenital malformations and development anomalies (CMDAs) without chromosome diseases. There were no

\footnotetext{
${ }^{1}$ HLA Alleles Numbers. Nomenclature. Available at: http://hla.alleles.org/nomenclature/stats.html (12.06.2020) (in Russian).
} 
children with CHDs in this group. All congenital malformations were sporadic without any traces in family case history. These malformations included the following: 25 children had III-IV degree hydrocephaly; 16 children, vascular plexus cysts; 114 children, hydronephrosis II; 5 children, Arnold-Chiari malformation; 5 children, vermis agenesis; and 3 children, one kidney agenesis. HLA-DRB1 allele combinations were analyzed in the same manner and totally we detected 544 combinations $(68 \cdot 8=544)$. This group was created at clinics within the $\mathrm{Ke}$ merovo State Medical University system.

Our reference group included 132 families who had two or more healthy children. 1,056 combinations were detected in this group $(132 \cdot 8=1,056)$; the group was created at ambulatories and polyclinics within the Kemerovo State Medical University system.

Additionally, 51 girls and 89 boys were examined at the Biological Faculty of the Kemerovo State University; they were all in their reproductive age and there were no blood relatives or married couples among them. These young people took part in an experiment entitled «HLA-associated olfactory selection». The research was approved by the local ethical committee at the Kemerovo State University and all people who took part in it gave their informed written consent. This group was used in the present research for calculating a probability that female and male $H L A-D R B 1$ alleles would meet in a reproductive population (random selection). This probability was calculated via multiplying a selected female allele by all male alleles in turn and vice versa. The calculation was performed for all female and male alleles. When transforming share values into absolute ones, we used an overall number of all possible combinations that was equal to $18,156(51 \cdot 89 \cdot 4=18,156)$.

Our research object was genome DNA extracted from peripheral blood leucocytes via phenol-chloroform extraction as per a conventional procedure. HLA-DRB1 typing was performed via PCR taking into account results obtained in real time mode with DT-96 detecting amplifier (DNA-technology, Russia). We determined frequency of 14 HLA-DRB1 gene alleles with commercial reagent sets HLADNA-TEX (Catalogue number R1-H001-S3/5, DNA-technology, Russia).

Research procedure. At the first stage in our research we compared frequencies of actual HLA-DRB1 allele combinations in the reference group and calculated combinations obtained for young males and females who were not blood relatives. Calculated combinations were also compared with actual HLA-DRB1 allele combinations in both test groups. Then we compared frequencies of HLA-DRB1 allele combinations in the reference group and two test ones. In addition, we compared frequencies of HLA-DRB1 allele combinations in families who had children with CHDs and those with children who had CMDAs.

At the next stage we analyzed how children inherited alleles from their parents in two test groups and the reference one. In the reference group we examined inheritance by healthy children, and in test groups, by children with CHDs and CMDAs respectively. For each HLA-DRB1 we took into account alleles that were inherited by children from mothers, fathers or both parents. If inheritance is equiprobable, there shouldn't be any significant differences between inherited and non-inherited alleles and delta between them should tend to zero. The first stage in the research focused on determining peculiarities of HLA assortativity in case a fetus was healthy and in case there was congenital fetus pathology. The second stage concentrated on revealing pre-natal selection, including gametogenesis and interaction as per HLA within «mother - fetus» system in case a fetus was healthy and in case congenital fetus malformation occurred.

All the data were mathematically processed with STATISTICA 8.0 (Stat Soft Inc., USA). Hardy-Weinburg equilibrium was determined with Pearson's chi-square. Expected frequencies (in shares) of allele combinations in case of random selection between people with different sexes were calculated via multiplying frequencies (in shares) of respective female alleles with male ones. Expected homozygocity was calculated via squaring a 
share of a respective allele. To reveal differences in frequencies of allele combinations in the examined groups and in inheritance from mothers to children, we used Pearson's chisquare with Yates's correction for continuity. To assess risks of a congenital heart disease, we calculated odds ratio $(O R)$ and its $95 \%$ confidence interval $(C I)$. Differences were considered statistically significant in all cases when $p<0.05$ [14].

Results and discussion. Our research revealed that there were no statistically significant differences between HLA-DRB1 genotype frequency and frequency of genotypes calculated with Hardy-Weinburg equilibrium.

Data given in Table 1 show that there were no differences in HLA-DRB1 alleles frequency between the group made up of young males and females where random allele combinations were estimated, and all the other groups made up of families; it indicates that the examined groups were comparable in terms of allele combinations.

We determined that frequency of HLA$D R B 1 * 12$ allele was higher among parents who had children with CMDAs against the reference group. Besides, this allele was more frequent in families who had children with CMDAs. We didn't detect any other statistically significant differences.

Then, according to the research procedure, we compared actual HLA-DRB1 allele combinations in the reference group and both test groups with calculated combinations obtained for young males and females who were not married or blood relatives (random selection). We revealed certain statistically significant differences that are given in Table 2.

Data given in Table 2 indicate that actual frequencies of allele combinations in families with healthy children were different from calculated ones (random selection) predominantly as per $H L A-D R B 1 * 01$ and $H L A-D R B 1 * 15$. Besides, positive selection involves a growing number of families with female and male HLA-DRB1*01, HLA-DRB1*15, HLA-DRB1*07 and HLA-DRB1*03(17), HLA-DRB1*4, HLA$D R B 1 * 15$ accordingly.

We analyzed deviations from random selection in families where children suffered from either CMDAs or CHDs; our analysis revealed the following. Only positive selection was detected in families where children had CMDAs and this selection involved married couples with female and male HLA$D R B 1 * 12$, as well as with female $H L A$ $D R B 1 * 12$ and male HLA-DRB $1 * 01, H L A-$ $D R B 1 * 04, \quad H L A-D R B 1 * 13, H L A-D R B 1 * 15$. Besides, just as it was the case with the reference group, there was positive selection in this group regarding a combination of female $H L A-D R B 1 * 07$ and male $H L A-D R B 1 * 15$ in a married couple.

Table 1

Distribution of $H L A-D R B 1$ alleles in the examined groups (\%)

\begin{tabular}{|c|c|c|c|c|c|}
\hline Alleles & 1. Random selection $(n=280)$ & 2. Reference $(n=528)$ & 3. CMDAs $(n=134)$ & 4. CHDs $(n=192)$ & $p$ \\
\hline 01 & 21.78 & 14.39 & 13.80 & 12.13 & 0.05 \\
\hline $03(17)$ & 7.85 & 9.47 & 2.23 & 10.46 & 0.05 \\
\hline 04 & 9.64 & 11.93 & 7.46 & 13.38 & 0.05 \\
\hline 07 & 7.50 & 9.28 & 10.07 & 11.29 & 0.05 \\
\hline 08 & 5.35 & 6.43 & 2.98 & 2.92 & 0.05 \\
\hline 09 & 0.71 & 4.16 & 1.86 & 0.83 & 0.05 \\
\hline 10 & 0.71 & 0.18 & 0.74 & 2.09 & 0.05 \\
\hline 11 & 11.42 & 10.98 & 15.67 & 15.48 & 0.05 \\
\hline 12 & 5.71 & 5.49 & 13.06 & 2.09 & $p_{2.3}=0.003$. \\
\hline 13 & 13.92 & 9.84 & 14.55 & 13.38 & 0.0007 \\
\hline 14 & 1.42 & 2.84 & 2.23 & 2.09 & 0.05 \\
\hline 15 & 11.07 & 11.74 & 14.17 & 13.80 & 0.05 \\
\hline 16 & 2.85 & 3.22 & 1.11 & 0.00 & 0.05 \\
\hline
\end{tabular}


Allele combinations in the randomly selected group (calculated values) and in married couples in the reference and test groups (only statistically significant differences, \%)

\begin{tabular}{|c|c|c|c|}
\hline A combination of a female /male allele & Random selection, $n=18,156$ & Reference, CMDAs, CHDs & $p$ \\
\hline \multicolumn{5}{|c|}{ Reference, $n=1,056$} \\
\hline $01 / 01$ & 4.73 & $1.52(-)$ & 0.01 \\
\hline $01 / 15$ & 2.15 & $5.21(+)$ & 0.008 \\
\hline $03(17) / 15$ & 0.94 & $3.03(+)$ & 0.007 \\
\hline $07 / 15$ & 0.61 & $2.75(+)$ & 0.004 \\
\hline $15 / 01$ & 2.54 & $5.41(+)$ & 0.009 \\
\hline $15 / 03(17)$ & 0.58 & $2.75(+)$ & 0.003 \\
\hline $15 / 04$ & 1.04 & $3.69(+)$ & 0.005 \\
\hline $15 / 15$ & 1.16 & $8.62(+)$ & 0.0001 \\
\hline \multicolumn{5}{|c|}{ CMDAs, $n=544$} & $2.57(+)$ & 0.006 \\
\hline $07 / 15$ & 0.61 & $2.39(+)$ & 0.004 \\
\hline $12 / 01$ & 0.48 & $1.47(+)$ & 0.003 \\
\hline $12 / 04$ & 0.21 & $4.41(+)$ & 0.001 \\
\hline $12 / 12$ & 0.26 & $1.84(+)$ & 0.002 \\
\hline $12 / 13$ & 0.29 & $2.21(+)$ & 0.001 \\
\hline $12 / 15$ & 0.22 & $2.64(+)$ & 0.03 \\
\hline \multicolumn{5}{|c|}{ CHDs, $n=384$} & $1.44(+)$ & 0.01 \\
\hline $04 / 04$ & 0.89 & $2.41(+)$ & 0.03 \\
\hline $07 / 03(17)$ & 0.31 & $1.44(+)$ & 0.008 \\
\hline $13 / 14$ & 0.79 &
\end{tabular}

$\mathrm{N}$ o t e : / is an allele combination given as follows: the first allele is a female one, the second, male. Signs (-) and $(+)$ indicate either negative or positive selection.

Results in families with children suffering from sporadic CHDs without chromosome diseases also deviated from random selection as there was positive selection for female and male allele $H L A-D R B 1 * 04$. Other significant differences from the reference group were detected in this group regarding frequency of allele combinations for female $H L A-D R B 1 * 07$ and male $H L A-D R B 1 * 03, H L A-D R B 1 * 13$ as well as female HLA-DRB1*13 and male HLA-DRB1*14.

The most significant differences between the reference group and the groups with CMDAs and CHDs were detected when frequencies of allele combinations were compared. As it is shown in Table 3, family homology as per $H L A-D R B 1 * 12$ was more frequent in families having children with CMDAs against the reference group, and homology as per HLA$D R B 1 * 15$ was less frequent.

These data indicate that $H L A-D R B 1 * 12$ allele might be a marker for a cohort with
CMDAs. It is quite possible that these peculiarities are related to a deviation from physiological assortativity as per $H L A-D R B 1 * 12$ when a new married couple is created and it happens among other things, due to impacts exerted by social, economic, macro- and micro-ecological factors.

Just as it was the case with families where children had CMDAs, negative selection was detected in the group where children had CNDs for female and male HLA-DRB $1 * 15$ and additionally for female $H L A-D R B 1 * 13$ and male HLA-DRB1*15. Female HLA$D R B 1 * 03(17), H L A-D R B 1 * 13$ and male HLA$D R B 1 * 04$ and $H L A-D R B 1 * 07$ respectively were more frequent in married couples in this group than in the reference one. One can see from the table that male alleles $H L A-D R B 1 * 04$ and $H L A-D R B 1 * 07$ were more frequent in married couples both in the group with CMDAs and with CHDs than in the reference 
Table 3

Allele combinations in families with healthy children and in families with children suffering from congenital malformations and development anomalies and congenital heart diseases

(only significant differences are given, \%)

\begin{tabular}{|c|c|c|c|}
\hline A combination of a female /male allele & Reference, $n=1,056$ & CMDAs, CHDs & $p$ \\
\hline \multicolumn{3}{|c|}{ CMDAs, $n=544$} \\
\hline $12 / 07$ & 0.27 & $2.21(+)$ & 0.02 \\
\hline $12 / 12$ & 0.82 & $4.41(+)$ & 0.009 \\
\hline $13 / 07$ & 0.27 & $3.68(+)$ & 0.01 \\
\hline $15 / 04$ & 3.69 & $0.37(-)$ & 0.008 \\
\hline $15 / 15$ & 8.62 & 0.001 \\
\hline \multicolumn{4}{|c|}{ CHDs, $n=384$} \\
\hline $3(17) / 04$ & 0.19 & $1.1(-)$ & 0.03 \\
\hline $13 / 07$ & 0.27 & $2.88(+)$ & 0.01 \\
\hline $13 / 15$ & 4.67 & $0.96(-)$ & 0.02 \\
\hline $15 / 15$ & 8.62 & $1.68(-)$ & 0.009 \\
\hline \multicolumn{7}{|c|}{ CMDA,,$n=544$} & 0.24 & 0.01 \\
\hline $12 / 12$ & $4.41(+)$ & $0.88(+)$ & 0.03 \\
\hline
\end{tabular}

$\mathrm{N}$ o t e : / is an allele combination given as follows: the first allele is a female one, the second, male. Signs (-) and $(+)$ indicate either negative or positive selection.

one. Special attention should be paid to positive selection of a family $H L A-D R B 1 * 17$ and $H L A-D R B 1 * 04$ allele combination in the group with children suffering from CHDs. This heterozygote type, just as HLA-DRB $1 * 04$, $H L A-D R B 1 * 03(17)$ alleles separately, is associated with multiple autoimmune diseases including insulin-dependent pancreatic diabetes $[15,16]$. Therefore, deviations occurring in the test groups when selection is taking place may create preconditions for immune-inflammatory pathology including reproductive disorders.

Having compared two test groups, with CMDAs and CHDs, we revealed that HLADRB1*12, HLA-DRB1*15 u HLA-DRB1*07 alleles were differentiating markers between them. Family homology as per HLA-DRB $1 * 12$ created statistically significant difference between CVDAs and CHDs groups.

Deviations from random HLA-DRB1 combinations in married couples and further euqiprobable inheritance can influence HLA$D R B 1$ allele distribution among healthy and sick children. We compared HLA-DRB1 allele frequency in the reference and both test groups and revealed alleles that were associated with pathology (Table 4). Thus, there were statisti- cally significant differences in frequency for three $H L A-D R B 1 * 03(17), \quad H L A-D R B 1 * 12$, HLA-DRB $1 * 15$ alleles.

$H L A-D R B 1 * 12$ allele turned out to be positively associated with CMDAs $(O R=5.72$; CI 95\% 2.25-14.42; $p<0.0001)$. HLA-DRB1*15 allele turned out to be the same for children with CHDs $(O R=1.83$; CI 95\% 0.73-4.71; $p=0.03)$. Differences as per this allele were detected only against the reference group but not against the group with CMDAs. Negative association with CMDAs was detected for $H L A-D R B 1 * 03(17) \quad(O R=0.13 ; \quad C I \quad 95 \%$ $0.05-0.32 ; p=0.0008)$. Authentic differences as per its frequency were detected for the groups with CMDAs and CHDs.

Our research revealed that there was a deviation from basic population selection in the groups with CHDs and CMDAs. We revealed not only a deviation from calculated homozygosity for the CHDs group but also an authentic increase in frequency of common HLA$D R B 1 * 04$, female $H L A-D R B 1 * 07$ with male $H L A-D R B 1 * 13, H L A-D R B 1 * 17$ and female $H L A-D R B 1 * 13$ with male HLA-DRB $1 * 14$ in married couples. Besides, certain alleles were more frequent in married couples from the CHDs group against the reference one. Given 
Allele frequency in children from the examined groups

\begin{tabular}{|c|c|c|c|c|c|c|c|}
\hline \multirow{2}{*}{ Allele } & \multicolumn{2}{|c|}{ 1. Reference, children } & \multicolumn{2}{c|}{ 2. CMDAs, children } & \multicolumn{2}{c|}{ 3. CHDs, children } & \multirow{2}{*}{$p$} \\
\cline { 2 - 7 } & total $(n=264)$ & $\%$ & total $(n=136)$ & $\%$ & total $(n=96)$ & $\%$ & 0.05 \\
\hline 01 & 35 & 13.26 & 10 & 7.46 & 14 & 9.93 & \multirow{2}{*}{$p_{1.2}=0.0008 . p_{2.3}=0.004$} \\
\hline $03(17)$ & 37 & 14.02 & 2 & 1.49 & 15 & 10.64 & 0.05 \\
\hline 04 & 32 & 12.12 & 13 & 9.70 & 20 & 14.18 & 0.05 \\
\hline 07 & 25 & 9.47 & 10 & 7.46 & 20 & 14.18 & 0.05 \\
\hline 08 & 15 & 5.68 & 2 & 1.49 & 5 & 3.55 & 0.05 \\
\hline 09 & 10 & 3.79 & 1 & 0.75 & 1 & 0.71 & 0.05 \\
\hline 10 & 2 & 0.76 & 1 & 0.75 & 2 & 1.42 & 0.05 \\
\hline 11 & 34 & 12.88 & 24 & 17.91 & 24 & 17.02 & 0.05 \\
\hline 12 & 14 & 5.30 & 33 & 24.63 & 2 & 1.42 & $p_{1.2}<0.0001 . p_{2.3}=0.0001$ \\
\hline 13 & 24 & 9.09 & 15 & 11.19 & 10 & 7.09 & 0.05 \\
\hline 14 & 7 & 2.65 & 3 & 2.24 & 3 & 2.13 & $\mathrm{p}_{1.3}=0.03$ \\
\hline 15 & 24 & 9.09 & 19 & 14.18 & 22 & 15.60 & 0.05 \\
\hline 16 & 5 & 1.89 & 1 & 0.75 & 3 & 2.13 & \\
\hline
\end{tabular}

$\mathrm{N}$ o t e : $n$ is the total number of alleles in children, * means $p<0.05$.

that, we can state that married couples that in future would have children with sporadic CHDs without chromosome diseases were created under influence exerted by additional social or biological factors that resulted in their deviation from physiological biological assortativity as per HLA (deviation from random selection) and physiological social one (deviation from the reference group). This assumption is well in line with significant associations between sporadic CHDs in children and their parents' medical and social factors including their mutual feelings and caring about each other [17]. L.I. Korochkin notes that early ontogenesis in a wider sense begins long before actual fertilization and embryo-fetogenesis and such events as a married couple being created and gametogenesis can rightfully be called «pre-adaptive» processes that reflect stages in early ontogenesis [18]. At this stage in ontogenesis people do not make their choice on a future spouse randomly and this stage can become a key one in health or diseases of the next generation. It was proven that HLA locus played a certain role in determining assortative selection related to olfactory response to pheromone smells [19].

It is quite possible that occurrence of married couples with their children suffering from CHDs was assortative as per poor or good education, intellect, welfare, and other social factors [17]. Bearing in mind that HLA-DRB1 alleles are associated with immune-inflammatory diseases, we should pay attention to married couples in this group tending to have common $H L A-D R B 1 * 04$. Multiple research works have revealed that this allele is associated with such immune pathologies as rheumatoid arthritis, insulin-dependent pancreatic diabetes, psoriasis, and other diseases [13, 20, 21]. There is an opinion that presentation of antigens by HLA-DR molecule coded by this allele involves apparent T-helpers activation including partial auto-orientation. If we apply this statement on immune response to autoand allo-antigens within «mother-embryo / fetus» system, we can assume that inflammatory process is decompensated within this system and a heart disease develops in an embryo as inflammatory embryopathy. We also revealed more frequent (against the reference group) female $H L A-D R B 1 * 03(17)$ and male $H L A-D R B 1 * 04$ combination in this group of families. HLA-DRB1*03(17) allele is associated with such immune pathologies as systemic lupus erythematosus, insulin-dependent pancreatic diabetes, bronchial asthma, etc. [20, 21]. Given that, such families have high risks of inflammatory embryopathy including those resulting in congenital heart diseases.

Meiotic drive is the second stage in population selection; it is a non-random selection of 
gametes participating in fertilization that occurs due to asymmetric division during ovogenesis and spermatogenesis. Selection during fertilization is also admissible; it happens due to sperms being tropic to ovicells with certain HLA haplotype sets [7]. Immune interaction occurring between a semi-allogenic embryo and a mother's immune medium is a vital stage in selecting HLA inheritance from parents to their children [22]. It was proven that embryos bearing father's HLA antigens that were different from mother's antigens (histoincompatible pregnancy) had selective advantage in their survival against embryos with their father's HLA being identical to mother's ones (histo-compatible pregnancy) [12].

We should note that sensitivity or resistance to infectious and parasitic agents produces selective effects regarding HLA polymorphism. Thus, polymorphism occurrence in antigen-identifying sites of HLA molecules, classes I and II, is related to natural selection associated with infectious, parasitic, micro- and macro-environment [7]. Heterozygote preferences that are put into effect via over-dominant selection of HLA alleles resistant to infectious agents are of great importance. Frequency-dependent selection imposes certain limitations on growing population heterozygocity. Bearing in mind that immune identification of a pathogen is controlled with HLA, experts manages to obtain empiric evidence of frequency-dependent selection that was put into effect in such a way that specific HLA haplotypes were resistant to certain infectious agents and sensitive to other ones at the same time [7]. We should note that associated sensitivity and resistance to infectious agents begins at fetal age when a fetus comes into contacts with residential viruses and mother's microbiome.
All the above-mentioned allows us to interpret our results stating that there was homology as per $H L A D R B 1 * 12$ in families where children had CMDAs; as per female $H L A D R B 1 * 15$ and male HLADRB ${ }^{*} 07$, in families where children had CHDs. Thus, as per data take form literature [23] HLADRB1*12 is associated with sensitivity to herpetic viruses, and $H L A D R B 1 * 15$, opportunistic pathogens activation and occurrence of humoral adaptive immune responses to them as per IgG and $\mathrm{IgE}$ types (infectious-allergic process) [24]. Accordingly, a certain contribution is made into CMDAs occurrence by activation of residential virus genomes including those existing within «mother-embryo/fetus» system; CHDs occur due to, among other things, effects produced by opportunistic pathogens in female reproductive tracts.

Conclusion. HLADRB $1 * 12$ and $H L A D R B 1 * 15$ alleles are candidate not only regarding creation of a new scenario for population selection in case of CMDAs and CHDs accordingly, but also risk markers indicating congenital anomalies might occur taking into account deviations from proper biological assortativity as per HLA.

Funding. The present research was accomplished due to support provided for a complex fundamental research program approved by the RAS Siberian Branch within the fundamental subject No. 0546-2019-0002 accepted by the Scientific Research Institute for Complex Issues of Cardiovascular Diseases and entitled «Pathogenetic substantiation for developing implants for cardiovascular surgery made of biologically compatible materials and implementing a patient-oriented approach using mathematic modeling, tissue engineering, and genome predictors».

Conflict of interests. The authors declare there is no any conflict of interests.

\section{References}

1. Petersdorf E.W., O'hUigin C. The MHC in the era of next-generation sequencing: Implications for bridging structure with function. Human immunology, 2019, vol. 80, no. 1, pp. 67-78. DOI: 10.1016/j.humimm.2018.10.002

2. Chen N., Wang W., Wang F., Dong L., Zhao S., Zhang W., Zhu F. The distributions of HLA-A, HLA-B, HLA-C, HLA-DRB1 and HLA-DQB1 allele and haplotype at high-resolution level 
in Zhejiang Han population of China. International journal of immunogenetics, 2019, vol. 46, no. 1, pp. 7-16. DOI: 10.1111/iji.12411

3. Vojvodić S.I., Ademović-Sazdanić D.S. Distribution of HLA DRB1, DQA1 and DQB1 Allelic Main Groups in the Vojvodina Province of Serbia: Genetic Relatedness with Other Populations. Russian Journal of Genetics, 2019, vol. 55, no. 1, pp. 124-130. DOI: 10.1111/j.1744-313X.2012.01122.x

4. Zhang X., Cheng Y., Zhang Q., Wang X., Lin Y., Yang C., Fan X. [et al.]. Meta-Analysis Identifies Major Histocompatiblity Complex Loci in or Near HLA-DRB1, HLA-DQA1, HLA-C as Associated with Leprosy in Chinese Han Population. Meta-Analysis, 2019, vol. 139, no. 4, pp. 957-960. DOI: 10.1016/j.jid.2018.09.029

5. Kiseleva A.N., Butina E.V., Isaeva N.V., Zaitseva G.A., Pozdeev N.M., Ovchinnikov V.V. Distribution of antigens of the HLA-system in married couples with reproductive disorders. Obstetrics, Gynecology and Reproduction, 2019, vol. 13, no. 2, pp. 111-118. DOI: 10.17749/2313-7347.2019.13.2.111-118

6. Tashiro R., Niizuma K., Khor S.S., Tokunaga K., Fujimura M., Sakata H., Tominaga T. Identification of HLA-DRB1*04: 10 allele as risk allele for Japanese moyamoya disease and its association with autoimmune thyroid disease: A case-control study. PloS One, 2019, vol. 14, no. 8, pp. e0220858. DOI: 0.1371/journal.pone.0220858

7. Makarchenko O.S., Gordeeva L.A., Shabaldin A.V., Glushkova O.A., Shatalina I.V., Simonova T.A., Filipenko M.L., Glushkov A.N., Kryukov P.M. Genes' immune presentation and immunoregulations role in forming conditions for fetus's losses. Mat' i ditya $v$ Kuzbasse, 2008, vol. 34, no. 3, pp. 13-20 (in Russian).

8. Lopes S.A.V.D.A., Guimarães I.C.B., Costa S.F.D.O., Acosta A.X., Sandes K.A., Mendes C.M.C. Mortality for critical congenital heart diseases and associated risk factors in newborns. A cohort study. Arquivos brasileiros de cardiologia, 2018, vol. 111, no. 5, pp. 666-673. DOI: 10.5935/abc.20180203

9. Kafian A.S., Mirshahi A., Amouzeshi A., Ramazani A.A., Bahman B., Hasanzadeh T.M., Salehi F. Epidemiologic Study of Congenital Heart Diseases and Its Related Factors in Children Referred to the Pediatric Cardiac Clinic of Birjand University of Medical Sciences, Iran. International Journal of Pediatrics, 2019, vol. 7, no. 12, pp. 10455-10463. DOI: 10.22038/ijp.2019.41467.3497

10. Aimagambetova G., Hajjej A., Malalla Z.H., Finan R.R., Sarray S., Almawi W.Y. Maternal HLA-DR, HLA-DQ, and HLA-DP loci are linked with altered risk of recurrent pregnancy loss in Lebanese women: A case-control study. American Journal of Reproductive Immunology, 2019, vol. 82, no. 4, pp. e13173. DOI: 10.1111/aji.13173

11. Emmery J., Hachmon R., Pyo C.W., Nelson W.C., Geraghty D.E., Andersen A.M.N., Hviid T.V.F. Maternal and fetal human leukocyte antigen class Ia and II alleles in severe preeclampsia and eclampsia. Genes \& Immunity, 2016, vol. 17, no. 4, pp. 251-260. DOI: 10.1038/gene.2016.20

12. Grimstad F., Krieg S. Immunogenetic contributions to recurrent pregnancy loss. Journal of assisted reproduction and genetics, 2016, vol. 33, no. 7, pp. 833-847. DOI: 10.1007/s10815-016-0720-6

13. Guseva V., Lapin S., Myachikova V., Maslyanski A., Chuchlovin A., Ivanova N., Tkachenko O., Blinova T., Totolian A. Clinical importance of determination of hla-drb1 locus genes in rheumatoid arthritis. Medical Immunology (Russia), 2019, vol. 21, no. 2, pp. 333-340. DOI: 10.15789/15630625-2019-2-333-340

14. Bland J.M., Altman D.G. The odds ratio. BMJ, 2016, vol. 320, no. 7247, pp. 1468. DOI: 10.1136/bmj.320.7247.1468

15. Nunes M.E.G., Rosa D.V., Fagundes E.D.T., Ferreira A.R., Miranda D.M.D., Ferri Liu P.M. HLA-DRB1 gene polymorphisms in pediatric patients with type 1 autoimmune hepatitis and type 1 autoimmune hepatitis overlap syndrome with autoimmune cholangitis. Arquivos de gastroenterologia, 2019, vol. 56, no. 2, pp. 146-150. DOI: 10.1590/S0004-2803.201900000-29

16. Tashiro R., Niizuma K., Khor S.S., Tokunaga K., Fujimura M., Sakata H., Tominaga T. Identification of HLA-DRB $1 * 04: 10$ allele as risk allele for Japanese moyamoya disease and its association with autoimmune thyroid disease: A case-control study. PloS One, 2019, vol. 14, no. 8, pp. e0220858. DOI: 10.1371/journal.pone.0220858

17. Yu D., Feng Y., Yang L., Da M., Fan C., Wang S., Mo X. Maternal Socioeconomic Status and the Risk of Congenital Heart Defects in Offspring: A Meta-Analysis of 33 Studies. PLoS ONE, 2014, vol. 9, no. 10, pp. e111056. DOI: 10.1371/journal.pone.0111056 
18. Korochkin L.I. Ontogenez, evolyutsiya i geny [Ontogenesis, evolution, and genes]. Priroda, 2002, no. 7, pp. 63-77 (in Russian).

19. Chuyanova A.A., Tsepokina A.V., Shabaldin A.V., Litvinova N.A., Zubrikova K.Yu., Boldyreva M.N. Features olfactory screening for HLA-DRB1 among unrelated donors of different sex. Immunologiya, 2015, vol. 36, no. 2, pp. 90-95 (in Russian).

20. Kular L., Liu Y., Ruhrmann S., Zheleznyakova G., Marabita F., Gomez-Cabrero D., Aeinehband S. [et al.]. DNA methylation as a mediator of HLA-DRB1*15: 01 and a protective variant in multiple sclerosis. Nature communications, 2018, vol. 9, no. 1, pp. 1-15. DOI: 10.1038/s41467-018-04732-5

21. Eltayeb-Elsheikh N., Khalil E., Mubasher M., Al Jurayyan A., AlHarthi H., Omer W.H., Elghazali G. Association of HLA-DR-DQ alleles, haplotypes, and diplotypes with Type 1 diabetes in Saudis. Diabetes/Metabolism Research and Reviews, 2020, pp. e3345. DOI: 10.1002/dmrr.3345

22. Erlebacher A. Immunology of the maternal-fetal interface. Annu. Rev. Immunol, 2013, vol. 31, pp. 387-411. DOI: 10.1146/annurev-immunol-032712-100003

23. Kovalic A.J., Bonkovsky H.L. The Pathogenesis of Autoimmune Liver Diseases. In Diagnosis and Management of Autoimmune Hepatitis, 2020, pp. 9-50.

24. Eidan A.J., AL-Harmoosh R.A., Hadi Z.J. Association of HLA-DRB1 Alleles with Allergic Asthma and Total Serum IgE Levels in Iraqi Adults Patients. Indian Journal of Public Health Research \& Development, 2020, vol. 10, no. 1, pp. 505-510. DOI: 10.5958/0976-5506.2019.00099.8

Shabaldin A.V., Tsepokina A.V., Dolgikh O.V., Shabaldina E.V., Ponasenko A.V. Combination of hla-drb1 alleles as a factor causing risks of sporadic congenital heart defects and congenital malformations without chromosome diseases. Health Risk Analysis, 2021, no. 1, pp. 133-142. DOI: 10.21668/health.risk/2021.1.14.eng

Received: 26.09 .2020

Accepted: 03.03.2021

Published: 30.03 .2021 